\title{
A Compact Dual-Band RFID Tag Antenna Mountable on Metallic Objects
}

\author{
Byeonggwi Mun, ${ }^{1}$ Yonghyun Yoon, ${ }^{1}$ Hyunwoo Lee, ${ }^{1}$ Hark-Yong Lee, ${ }^{2}$ and Byungje Lee \\ ${ }^{1}$ Department of Wireless Communications Engineering, Kwangwoon University, 20 Gwangun-ro, Nowon-gu, \\ Seoul 139-701, Republic of Korea \\ ${ }^{2}$ Sonovision Co., Ltd., Room 110, Seongnam Venture Building, 57 Talli-ro, Sujeong-gu, Seongnam-si, \\ Gyeonggi-do 461-804, Republic of Korea \\ Correspondence should be addressed to Byungje Lee; bj_lee@kw.ac.kr
}

Received 24 September 2014; Revised 9 December 2014; Accepted 10 December 2014

Academic Editor: Vincenzo Galdi

Copyright (C) 2015 Byeonggwi Mun et al. This is an open access article distributed under the Creative Commons Attribution License, which permits unrestricted use, distribution, and reproduction in any medium, provided the original work is properly cited.

\begin{abstract}
A compact $\left(50 \times 50 \times 4 \mathrm{~mm}^{3}\right)$ dual-band radio frequency identification (RFID) tag antenna mountable on metallic objects is proposed for the ultra-high frequency (UHF) band (917 923.5 MHz) and the microwave (MW) band (2.4 2.45 GHz). With the proximity-coupled feed loop, the proposed antenna consists of two symmetric planar inverted-F antenna (PIFA) elements for the UHF band passive tag and a meander microstrip patch antenna for the MW band active tag. The performance of the proposed antenna is verified by mounting it on the different sizes of the metallic object. Furthermore, the passive tag antenna in the UHF band furthermore may be used for energy harvesting techniques to improve the lifetime of the active tag in the MW band. The measured maximum read range is $5.50 \mathrm{~m}$ in the UHF band and $14.15 \mathrm{~m}$ in the MW band when the proposed tag antenna is mounted on the metallic objects. The total efficiency for all operating frequency bands is higher than $50 \%$. High isolation ( $>12 \mathrm{~dB})$ between tag antennas in the UHF band and the MW band is achieved.
\end{abstract}

\section{Introduction}

A radio frequency identification (RFID) system identifies an object by using short-range digital wireless techniques without direct contact. The number of applications for RFID systems has been growing rapidly in industries such as service, inventory control, distribution logistics, security systems, manufacturing process control, medical and biological, vehicular applications, and railway [1-4]. The RFID system consists of a reader and a tag that are separated by a short distance. Since they communicate with each other by electric/ magnetic field coupling or electromagnetic waves, the antenna is one of the key factors for RFID systems. The optimized antenna design of the RFID system can give a longer reading range, better accuracy, and reduced antenna fabrication cost.

When the reader emits a radio-frequency signal, it then detects and interrogates the tag for its content information, and the tag then sends a return signal back to the reader. Tags are usually small, simple devices consisting of an antenna and an IC chip. Tags can be classified as passive, active, or semiactive according to how they generate power when interrogated by the reader. While an active or semiactive tag has a battery to supply power, a passive tag uses the power emitted from the reader [5]. For an RFID active tag, since its operational lifetime is limited by the on-board battery, the need of long time PM (power management) techniques is one of the major areas [6]. The main goal for achieving a long lifetime is the optimization of the energy budget of the tag. This is however not possible using single use batteries without replacing them frequently. Thus, through energy harvesting techniques, additional energy sources may support the onboard battery.

The allocated frequency bands for RFID applications vary within a broad frequency range from low frequency (LF) $(120-140 \mathrm{kHz})$, high frequency (HF) $(13.56 \mathrm{MHz})$, and ultrahigh frequency (UHF) (868-928 MHz) to microwave (MW) $(2.45 \mathrm{GHz}$ and $5.8 \mathrm{GHz})$. The UHF band becomes attractive because of the high reading speed, capable multiple accesses, anticollision, and long reading distance compared to the 
LF and the HF bands. The MW band has been used for the industrial, scientific, and medical (ISM) band in the various radio telecommunication services [7]. It has similar electromagnetic characteristics as those of the UHF band.

To obtain a high reliability for reading range and reading rate and to achieve a long lifetime with additional energy harvesting techniques, dual-band operation is demanded for RFID tags [8-10]. Thus, determining how to design a compact dual-band antenna within a small volume can be the most important issue. For dual-band operation, passive and active tag antennas may be designed in the UHF and MW bands, respectively. An active tag in the MW band may then be able to harvest additional energy from a passive tag antenna in the UHF band by applying energy harvesting techniques, so that a long lifetime of an active tag can be achieved by supporting the on-board battery.

In general, the performance of the tag seriously affects the performance of the whole RFID system, while the antenna is the major part of the RFID system that mostly affects the ability to read the tag. The tag antenna thus needs to be designed considering its operating environments or platforms. When it is mounted on metallic objects, the antenna performance can be seriously decreased because of the reactance variation on the antenna impedance $[11,12]$. Particularly, for a passive UHF band RFID system, a tag does not contain its own power source. The reader antenna sends a radio signal to activate the passive tags, then listens for their backscatter, and reads the data they transmitted. A passive tag antenna must be designed to transmit the power efficiently, obtained from the reader antenna, to the microchip without possible losses. Therefore, impedance matching between the tag antenna and the microchip is very important. The impedance of an RFID tag antenna also varies when it is mounted on different objects. Particularly, metallic objects strongly affect the antenna performance by lowering the efficiency of a tag. Therefore, tag antennas must be designed to enable tags to be read near and on metallic objects without severe performance degradation. In order to achieve stable antenna performance on various metallic platforms, it is important to minimize the effect of the metallic supporting object.

In this paper, a compact $\left(50 \times 50 \times 4 \mathrm{~mm}^{3}\right)$ dual-band RFID tag antenna mountable on metallic objects without severe performance degradation is proposed for UHF band (917 923.5 MHz) and MW band $(2.4 \sim 2.45 \mathrm{GHz})$. With the proximity-coupled feed structure, two PIFA elements operate for a passive tag in the UHF band, and they furthermore may be used for energy harvesting techniques to improve a lifetime of an active tag in the MW band. A meander microstrip patch antenna in conjunction with the proximitycoupled feed structure operates for an active tag in the MW band. The proposed antenna has two $50 \Omega$ ports in order to be applicable for energy harvesting techniques to an active tag.

\section{Antenna Design and Results}

Figure 1 shows the geometry of the proposed antenna and detailed dimensions of the design parameters. To enable tags to be read on metallic objects without severe performance degradation and to be designed within a small volume, the proposed dual-band RFID tag antenna consists of two PIFA elements (Radiators 1 and 2), a meander microstrip patch (Radiator 3), a common ground plane, inductive coupled feed loops (Feeding loops 1 and 2), and shorting strips (Shorting strips 1 and 2) as shown in Figure 1(a). The overall size of the antenna including the ground plane is $50 \times 50 \times 4 \mathrm{~mm}^{3}$ as shown in Figure 1(b). Two PIFA elements and a meander microstrip patch operate in the UHF band and in the MW band, respectively.

Two $50 \Omega$ ports are available in the proposed dual-band RFID tag antenna so that an active tag in the MW band is possibly able to extend the lifetime by harvesting an additional energy from a passive tag antenna in the UHF band. Radiators and feeding loops are etched within the volume of $45 \times 45 \times 1 \mathrm{~mm}^{3}$ and on the FR4 $\left(\varepsilon_{r}=4.4\right)$ substrate as shown in Figures 1(a) and 1(b); and these are then finally mounted on the foam $\left(\varepsilon_{r}=1.1\right.$ and thickness $\left.=3 \mathrm{~mm}\right)$ with the common ground plane.

Several possible antenna types can be used for RFID tags. The dipole types of antennas such as folded dipoles and meandered dipoles are used in many applications since they can be printed on very thin film. The label-type tag antenna where the dipole is printed on a thin film has been used in many nonmetallic platforms. When they are however mounted on the metallic objects, the antenna performance is seriously deteriorated because of the reactance variation on the antenna impedance. To overcome this problem, several PIFAs, IFAs, or microstrip patch antennas have been proposed for tag antennas mountable on metallic objects [13-16]. The proposed dual-band tag antenna, which consists of two PIFA elements and a meander microstrip patch in conjunction with the proximity-coupled feed structure to broaden bandwidth, is thus designed as mountable on the metallic objects without severe performance degradation and within a small volume.

Two PIFA elements (Radiators 1 and 2), which are fed by the inductive coupling loop (Feeding loop 1) as shown in Figure 1, cover for the UHF band (917 923.5 MHz). Figure 2(a) shows the simulated surface current distributions on Radiators 1 and 2 at $920 \mathrm{MHz}$. They are in phase with equal amplitude, symmetrical with respect to the $y$-axis, and are inductively coupled to each other. At the open ends of two PIFA elements, the distance between Radiators 1 and 2 is about $1 / 4 \lambda$ at $900 \mathrm{MHz}$. E-field direction of the main beam is along the $y$-axis due to the symmetric current distribution on the radiators. The main beam direction of the proposed antenna is normal $(+z$-axis $)$ to the ground plane. Major coupling therefore comes from between the two symmetrical radiating PIFA elements rather than between the radiator and the ground plane. This means that the radiation of the proposed antenna at the UHF band comes mainly from these two PIFA elements, so that performance degradation of a tag antenna due to metallic objects can be considerably reduced. In order to obtain the better impedance match, two stubs are added into Feeding loop 1 by increasing the inductance between the two PIFA elements and the feeding loop structure. 

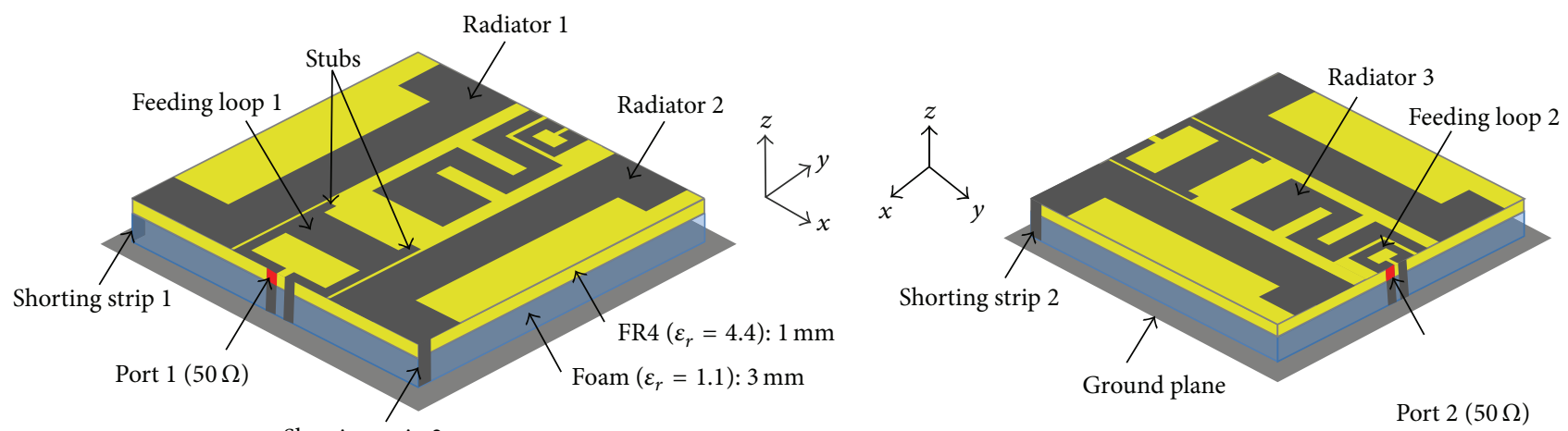

Shorting strip 2

(a)

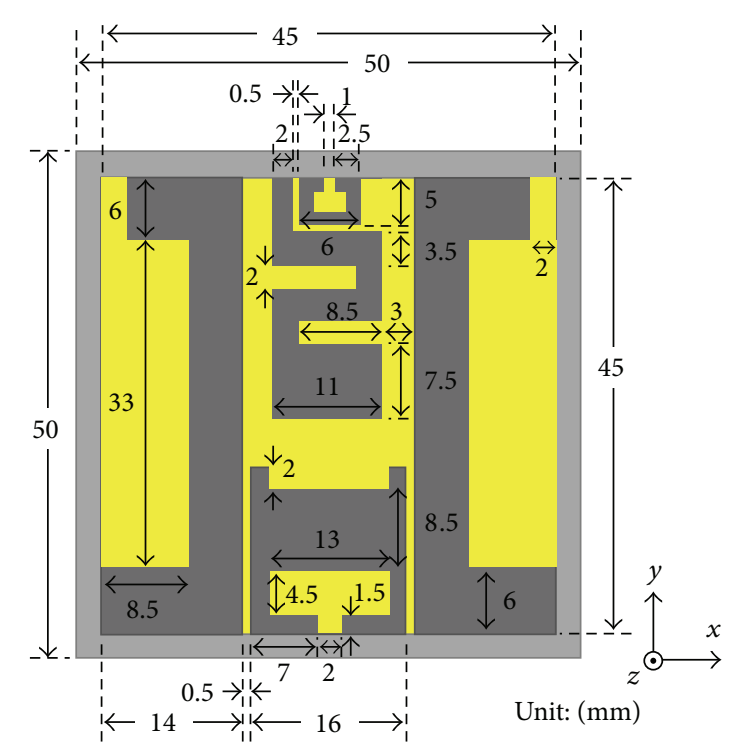

(b)

FIGURE 1: Structure of the proposed dual-band RFID tag antenna: (a) overall view and (b) top view with detailed dimensions.

The meander microstrip patch antenna (Radiator 3), which is fed by the inductive coupling loop (Feeding loop 2) to achieve wide impedance bandwidth as shown in Figure 1, covers the MW band $(2.4 \sim 2.45 \mathrm{GHz})$. Figure 2(b) shows the simulated surface current distribution on Radiator 3 at $2.425 \mathrm{GHz}$. E-field direction of the main beam is along the $y$ axis, and the main beam direction is normal $(+z$-axis $)$ to the ground plane. In order to obtain the more compact volume of a dual-band tag antenna, Radiator 3 is meandered between Radiators 1 and 2. Since the microstrip patch antenna has its own ground plane to operate, it may be a good choice for a RFID tag that identifies metallic objects. In general, a microstrip patch antenna has stable performance when its ground plane is extended more than $0.25 \lambda$ from the radiating patch. Therefore, the proposed microstrip patch antenna, which has a relatively larger $(50 \mathrm{~mm} \times 50 \mathrm{~mm}=0.83 \lambda \times 0.83 \lambda$ at $2.4 \mathrm{GHz}=0.31 \lambda \times 0.31 \lambda$ at $900 \mathrm{MHz}$ ) common ground plane in the MW band than in the UHF band, can give stable tag performance on metallic objects without severe performance degradation.
Figure 3 shows a photograph of the proposed antenna used for the measurement. When measuring the electric field pattern and total efficiency, Port 1 for the UHF band tag antenna was excited, while port 2 for the MW band tag antenna was terminated to a load with $50 \Omega$ and vice versa. Figure 4 shows the simulated and measured radiation patterns of the proposed antenna at $920 \mathrm{MHz}$ and at $2.425 \mathrm{GHz}$. It is noticed that the main beam directions both in the UHF band and in the MW band are normal ( $+z$-axis) to the ground plane. The simulated and measured radiation patterns agree well.

Figure 5 shows the simulated and measured $S$-parameters of the proposed dual-band RFID tag antenna. It shows that the proposed antenna covers both the UHF band (917 $923.5 \mathrm{MHz}$, VSWR < 2) and the MW band $(2.4 \sim 2.45 \mathrm{GHz}$, VSWR $<2$ ). Although the proposed antenna is designed within a compact size, high isolation $\left(S_{21}\right.$ and $\left.S_{12}>12 \mathrm{~dB}\right)$ between the UHF band tag antenna and the MW band tag antenna is achieved. Figure 6 shows the simulated and measured total efficiencies of the proposed antenna. It is 


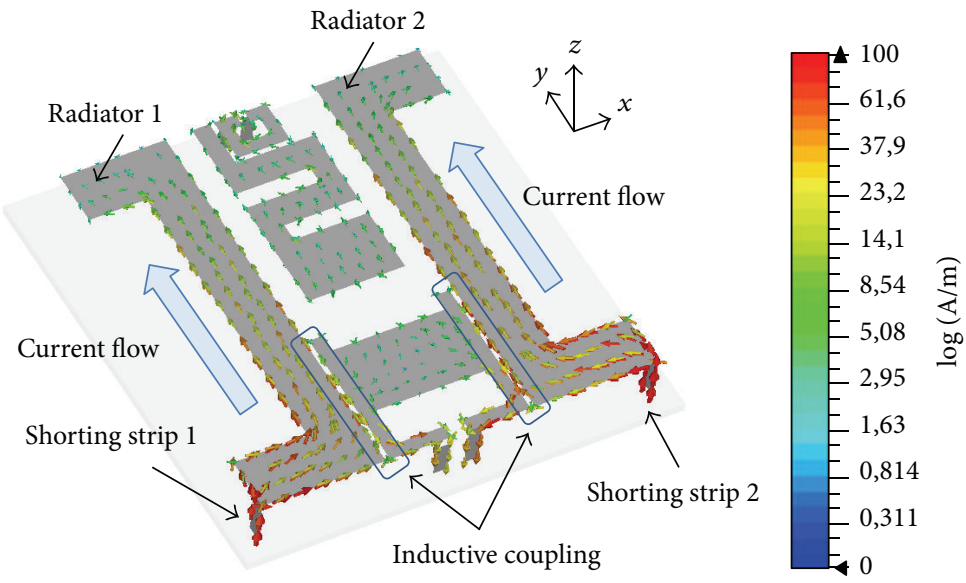

(a)

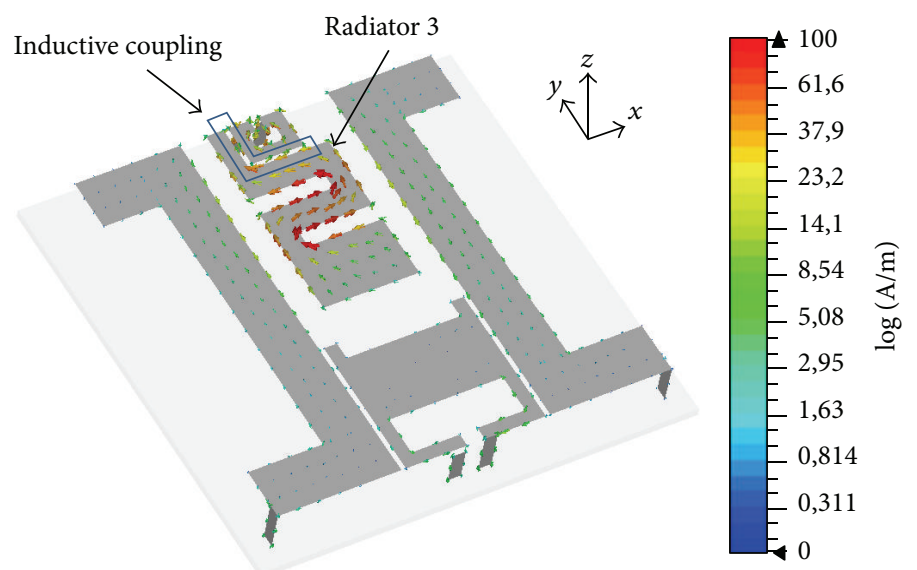

(b)

FIGURE 2: Simulated surface current distributions on the proposed antenna: (a) $920 \mathrm{MHz}$ and (b) $2.425 \mathrm{GHz}$.

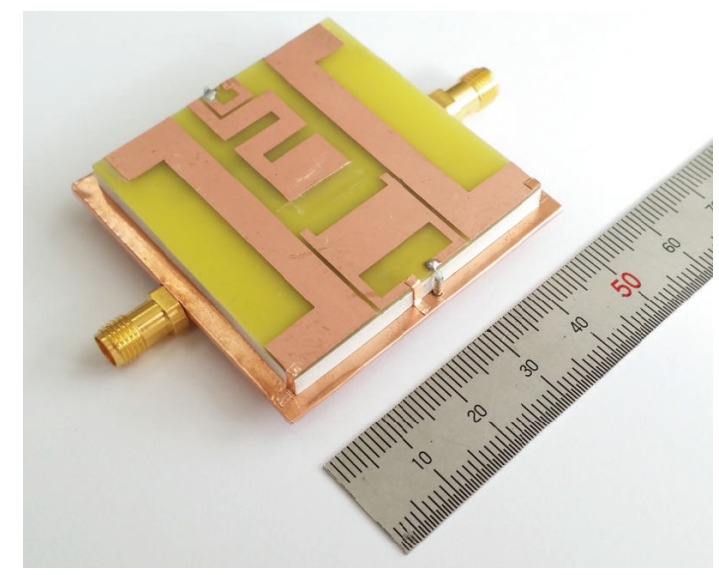

Figure 3: Photo of the fabricated antenna.

noticed that the proposed antenna gives relatively good efficiency $(>50 \%)$ for all operation frequency bands [17].

To study the effect of metallic objects, the performance of the proposed antenna is measured by mounting it on different sized metallic objects (copper plate). The size of the copper plate is varied from $0.5 \lambda \times 0.5 \lambda$ to $1 \lambda \times 1 \lambda$ at $900 \mathrm{MHz}$ in the UHF band, which is from $1.33 \lambda \times 1.33 \lambda$ to $2.67 \lambda \times 2.67 \lambda$ at $2.4 \mathrm{GHz}$ in the MW band. Figure 7 shows the measured $S$ parameter of the proposed antenna. It is noticed that the $S$ parameter of the proposed antenna is barely changed when 


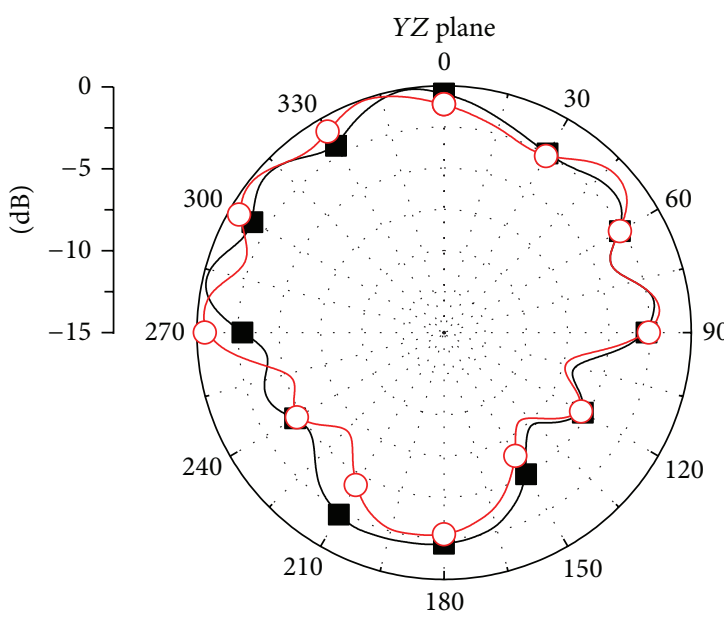
- - Simulated radiation pattern
$-\bigcirc-$ Measured radiation pattern

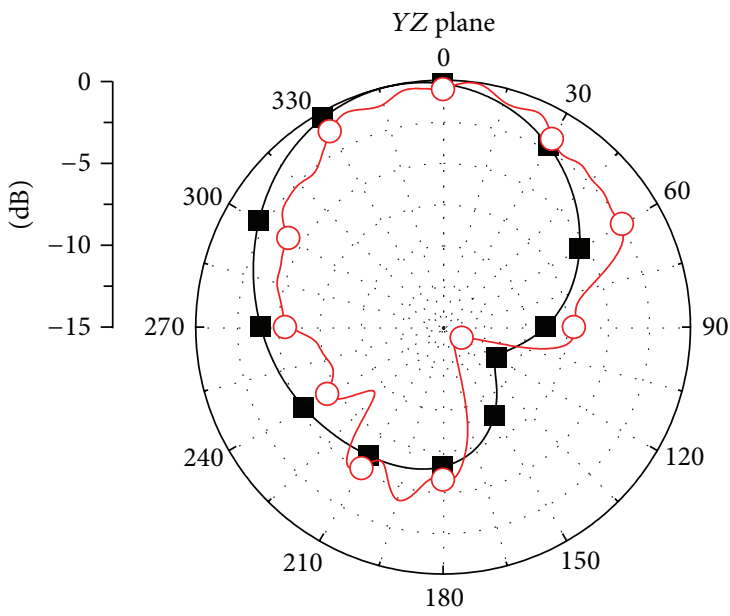

Simulated radiation pattern Measured radiation pattern

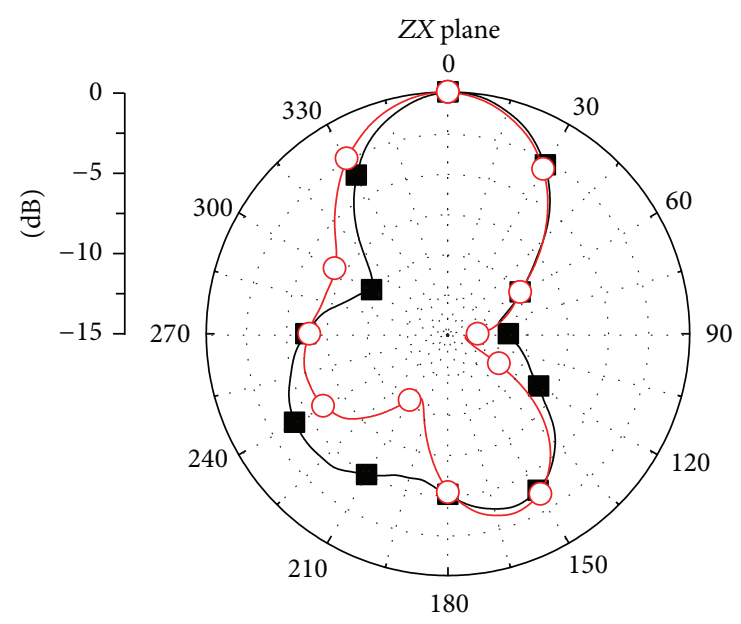

Simulated radiation pattern Measured radiation pattern

(a)
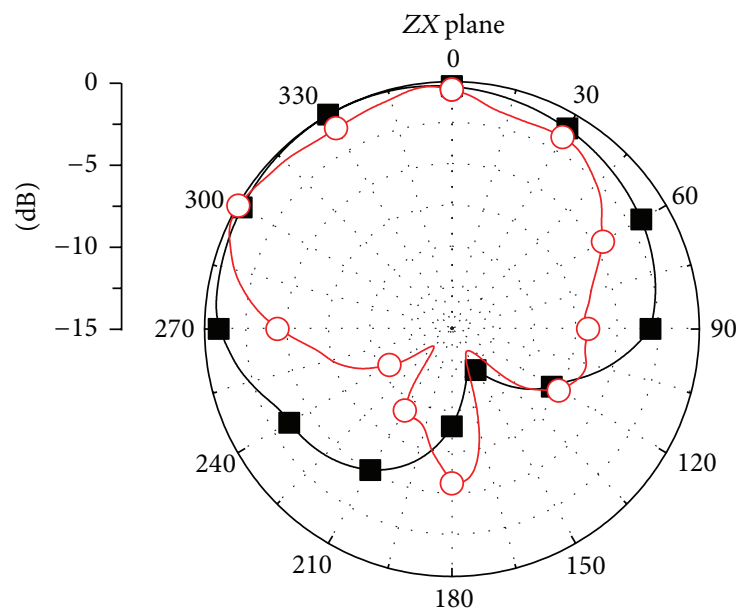

Simulated radiation pattern

Measured radiation pattern

(b)

FIGURE 4: Radiation pattern of the proposed antenna without metallic object: (a) $920 \mathrm{MHz}$ and (b) $2.425 \mathrm{GHz}$.

it is mounted on the different sized metallic objects. Figure 8 shows the measured total efficiency of the proposed antenna mounted on the metallic objects. It is noticed that when the proposed antenna is mounted on the metallic object, while total efficiency increases slightly more than that without the metallic object, it is almost identical for the different sizes of the metallic object. Since radiation of the proposed antenna in the UHF band comes mainly from two symmetrical PIFA elements rather than coupling between the radiator and the ground plane, a tag can thus give stable performance on the metallic objects without severe performance degradation. In addition, since the relatively larger electrical size $(1.33 \lambda \times$ $1.33 \lambda$ to $2.67 \lambda \times 2.67 \lambda$ at $2.4 \mathrm{GHz}$ ) of the metallic object in the MW band can be operated as the reflector for a microstrip patch antenna, it may improve efficiency. Figure 9 shows the measured radiation patterns at $920 \mathrm{MHz}$ in the UHF band and at $2.425 \mathrm{GHz}$ in the MW band. It is shown that when the proposed antenna is mounted on the different sizes of the metallic object, it gives a slightly higher directivity, and its back radiation is considerably reduced. Table 1 shows the measured read range of the proposed antenna with RFID IC (ATA5590) [18]. In the UHF band, the maximum read range is $5.15 \mathrm{~m}$ and $5.50 \mathrm{~m}$ without the metallic object and with the metallic object, respectively. In the MW band, the maximum read range is $13.50 \mathrm{~m}$ and $14.15 \mathrm{~m}$ without the metallic object and with the metallic object, respectively. The measured 
TABLE 1: Measured maximum read range with and without metallic objects.

\begin{tabular}{lcc}
\hline Operating bands & Measurement condition & $\begin{array}{c}\text { Maximum read } \\
\text { range }\end{array}$ \\
\hline UHF band & Without metallic object & $5.15 \mathrm{~m}$ \\
$(917 \sim 923.5 \mathrm{MHz})$ & $0.5 \lambda \times 0.5 \lambda$ copper plate & $5.50 \mathrm{~m}$ \\
& $1.0 \lambda \times 1.0 \lambda$ copper plate & $5.50 \mathrm{~m}$ \\
\hline MW band & Without metallic object & $13.50 \mathrm{~m}$ \\
$(2.4 \sim 2.45 \mathrm{GHz})$ & $1.33 \lambda \times 1.33 \lambda$ copper plate & $14.15 \mathrm{~m}$ \\
& $2.67 \lambda \times 2.67 \lambda$ copper plate & $14.15 \mathrm{~m}$ \\
\hline
\end{tabular}

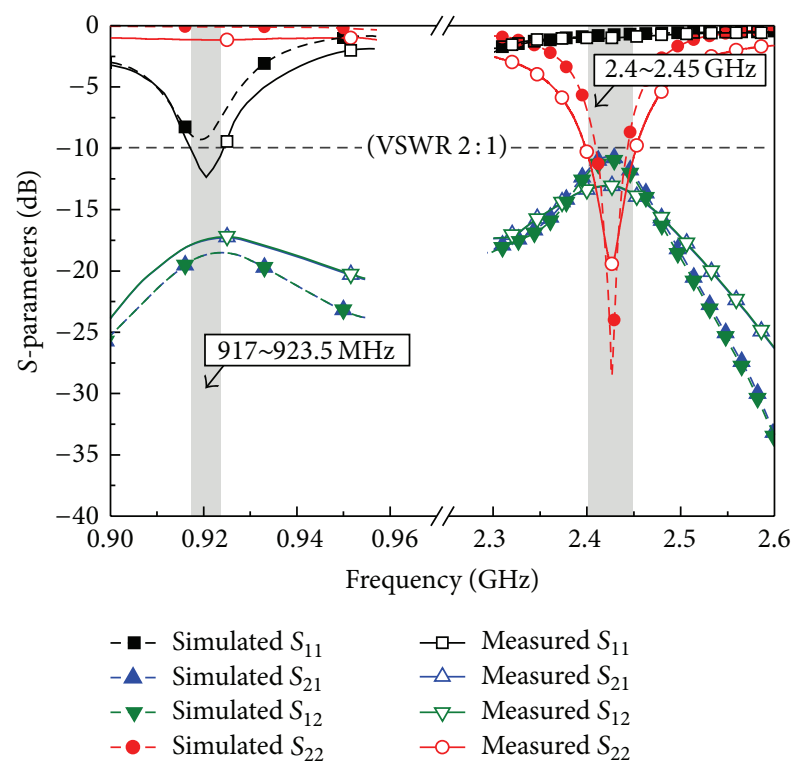

Figure 5: S-parameter of the proposed antenna without metallic objects.

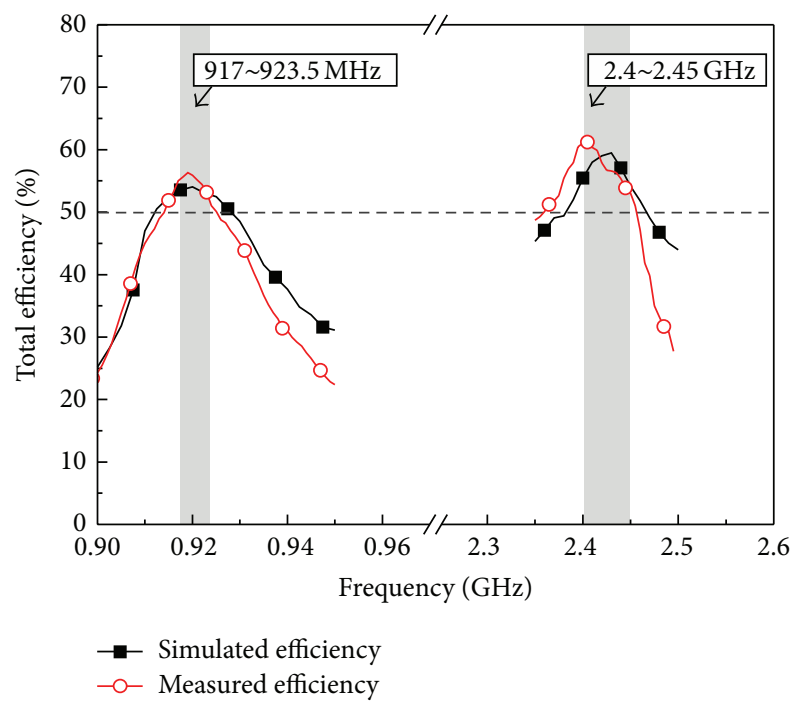

FIGURE 6: Total efficiency of the proposed antenna without metallic objects.

results show that the proposed antenna gives a slightly longer read when it is even mounted on various sizes of the metallic object.

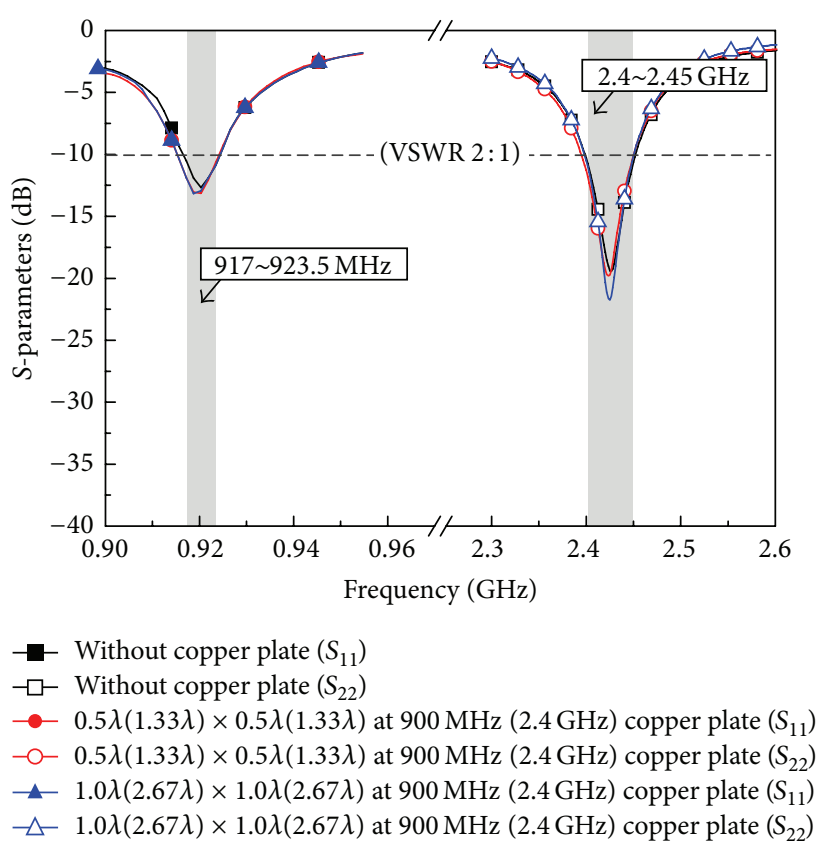

FIGURE 7: Measured S-parameters of the proposed antenna mounted on the different sized metallic objects.

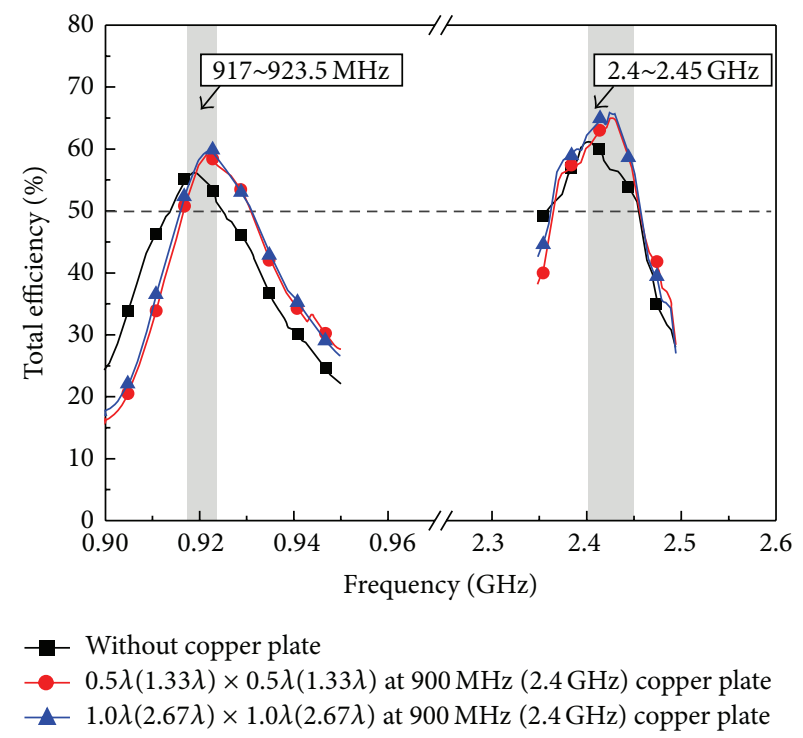

Figure 8: Measured total efficiency of the proposed antenna mounted on the different sized metallic objects.

\section{Conclusion}

A compact $\left(50 \times 50 \times 4 \mathrm{~mm}^{3}\right)$ dual-band RFID tag antenna is proposed for the UHF band $(917 \sim 923.5 \mathrm{MHz})$ and the MW band (2.4 2.45 GHz). By using two symmetrical PIFA elements in the UHF band and a meander microstrip patch in the MW band, in conjunction with the proximity-coupled feed structure to broaden bandwidth, the proposed antenna, which is mountable on metallic objects without severe performance degradation, can be designed within a compact volume. Having two $50 \Omega$ ports in the proposed antenna, a 

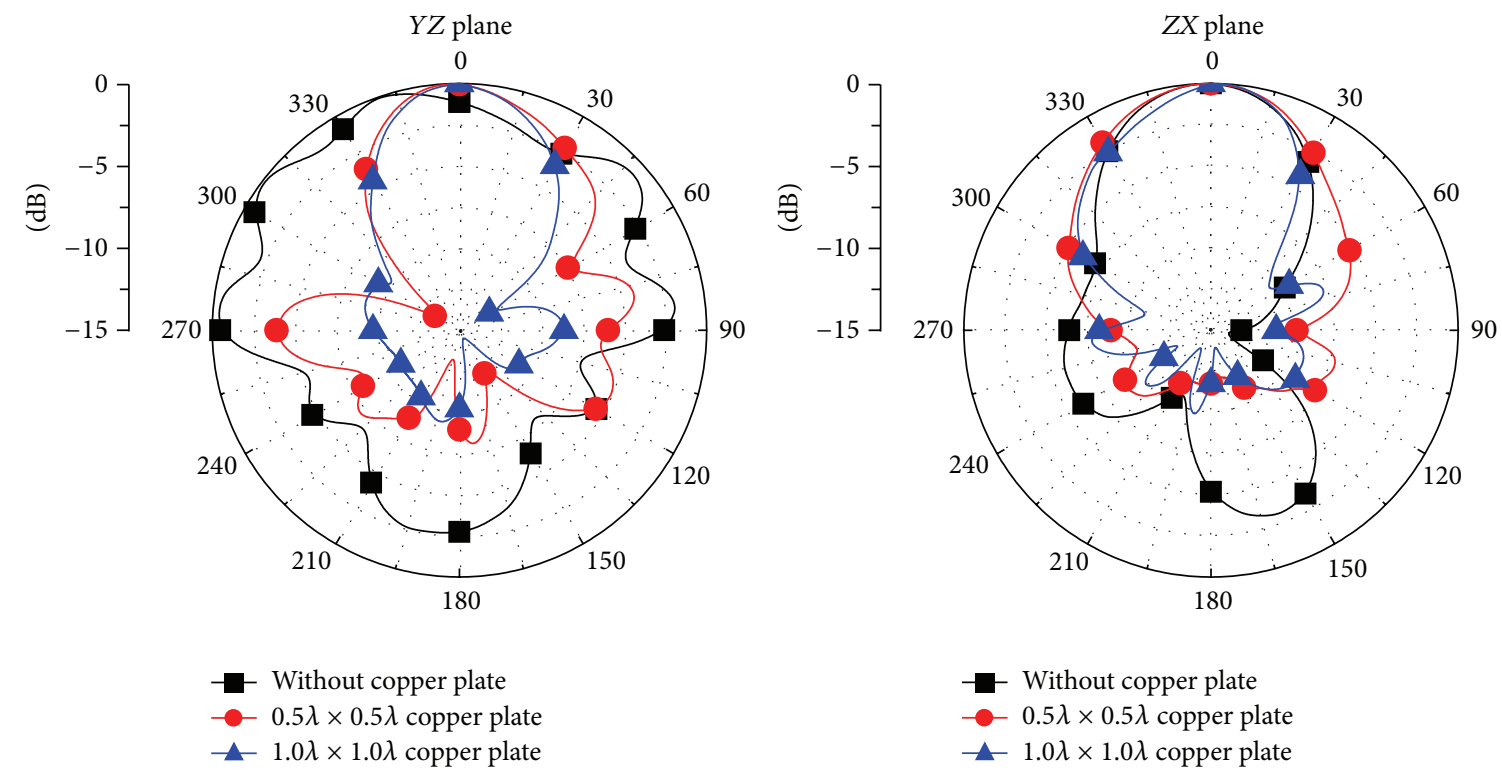

(a)
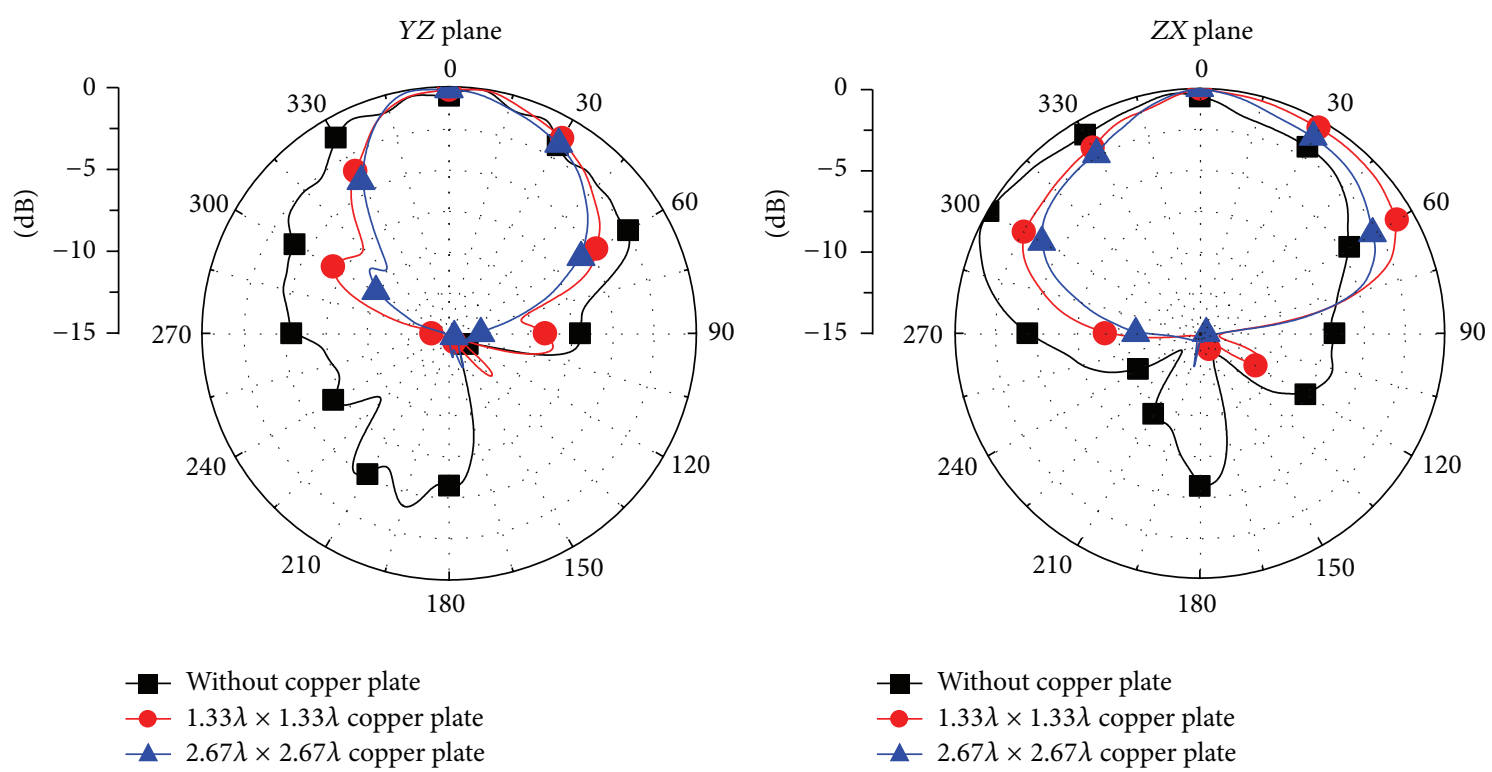

(b)

FiguRE 9: Measured radiation patterns of the proposed antenna mounted on the different sized metallic objects: (a) $920 \mathrm{MHz}$ and (b) $2.425 \mathrm{GHz}$.

passive tag antenna in the UHF band may furthermore be used for energy harvesting techniques to improve the lifetime of an active tag in the MW band. The total efficiency for all operating frequency bands is higher than $50 \%$. High isolation $(>12 \mathrm{~dB}$ ) between tag antennas in the UHF band and the MW band is achieved. The maximum read range is $5.50 \mathrm{~m}$ and $14.15 \mathrm{~m}$ in the UHF band and in the MW band, respectively, when the proposed RFID tag antenna is mounted on the metallic objects.

\section{Conflict of Interests}

The authors declare that there is no conflict of interests regarding the publication of this paper.

\section{Acknowledgment}

This work was supported by the ICT R\&D program of MSIP/IITP under the development of dual-band active RFID 
tag and application software for smart management of railways (Grants no. 10043982).

\section{References}

[1] L. Ukkonen, L. Sydanheimo, and M. Kivikoski, "Read range performance comparison of compact reader antennas for a handheld UHF RFID reader," in Proceedings of the IEEE International Conference on RFID, pp. 63-70, Grapevine, Tex, USA, March 2007.

[2] L. T. Lee and K. F. Tsang, "An active RFID system for railway vehicle identification and positioning," in Proceedings of the IET International Conference on Railway Engineering (ICRE '08), pp. 120-123, March 2008.

[3] D.-Y. Kim, H.-G. Yoon, B.-J. Jang, and J.-G. Yook, "Effects of reader-to-reader interference on the UHF RFID interrogation range," IEEE Transactions on Industrial Electronics, vol. 56, no. 7, pp. 2337-2346, 2009.

[4] X. Qing, Z. N. Chen, and C. K. Goh, "UHF near-field RFID reader antenna with capacitive couplers," Electronics Letters, vol. 46, no. 24, pp. 1591-1592, 2010.

[5] K. Finkenzeller, RFID Handbook: Radio-Frequency Identification Fundamentals and Applications, John Wiley \& Sons, London, UK, 2003.

[6] V. Pillai, H. Heinrich, D. Dieska, P. V. Nikitin, R. Martinez, and K. V. S. Rao, "An ultra-low-power long range battery/passive RFID tag for UHF and microwave bands with a current consumption of $700 \mathrm{nA}$ at 1.5 V,' IEEE Transactions on Circuits and Systems I: Regular Papers, vol. 54, no. 7, pp. 1500-1512, 2007.

[7] ITU-T, "Electromagnetic characterization of the radiated environment in the $2.4 \mathrm{GHz}$ ISM band," Telecommunication Standardization Sector of ITU Recommendation ITU-T K.79, 2009.

[8] H. Kimouche, H. Zemmour, and B. Atrouz, "Dual-band fractal shape antenna design for RFID applications," Electronics Letters, vol. 45, no. 21, pp. 1061-1063, 2009.

[9] W.-B. Zeng, X.-Y. Li, and J.-X. Liu, "A dual-band RFID slot tag antenna for ITS application," in Proceedings of the 3rd International Conference on Consumer Electronics, Communications and Networks (CECNet '13), pp. 5-7, November 2013.

[10] P. Jankowski-Mihułowicz, W. Kalita, M. Skoczylas, and M. Wȩglarski, "Modelling and design of HF RFID passive transponders with additional energy harvester," International Journal of Antennas and Propagation, vol. 2013, Article ID 242840, 10 pages, 2013.

[11] P. Raumonen, L. Sydänheimo, L. Ukkonen, M. Keskilammi, and M. Kivikoski, "Folded dipole antenna near metal plate," in Proceedings of the IEEE Antennas and Propagation Society International Symposium, pp. 848-851, June 2003.

[12] B. Yu, S.-J. Kim, B. Jung, F. J. Harackiewicz, and B. Lee, "RFID tag antenna using two-shorted microstrip patches mountable on metallic objects," Microwave and Optical Technology Letters, vol. 49, no. 2, pp. 414-416, 2007.

[13] L. Ukkonen, D. Engels, L. Sydanheirno, and M. Kivikoski, "Planar wire-type inverted-F RFID tag antenna mountable on metallic objects," in Proceedings of the IEEE Antennas and Propagation Society International Symposium, pp. 101-104, June 2004.

[14] L. Ukkonen, L. Sydänheimo, and M. Kivikoski, "A novel tag design using inverted-F antenna for radio frequency identification of metallic objects," in Proceedings of the IEEE/Sarnoff Symposium on Advances in Wired and Wireless Communication, pp. 91-94, Princeton, NJ, USA, April 2004.
[15] M. Hirvonen, P. Pursula, K. Jaakkola, and K. Laukkanen, "Planar inverted-F antenna for radio frequency identification," Electronics Letters, vol. 40, no. 14, pp. 848-850, 2004.

[16] Y. Yao, Y. Sui, X. Chen, and J. Yu, "Planar antenna for RFID tags on metal platform," in Proceedings of the International Workshop on Antenna Technology (iWAT '11), pp. 408-411, March 2011.

[17] M. Svanda and M. Polivka, "Small-size wearable high-efficiency tag antenna for UHF RFID of people," International Journal of Antennas and Propagation, vol. 2014, Article ID 509768, 5 pages, 2014.

[18] http://www.atmel.com/. 

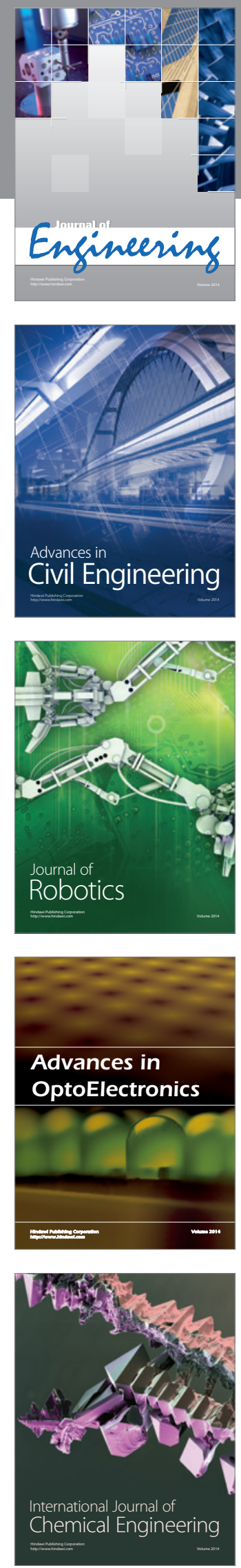

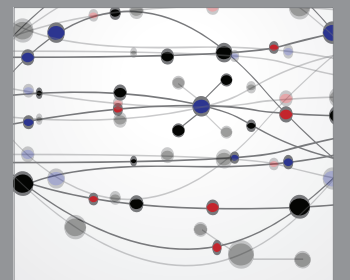

The Scientific World Journal
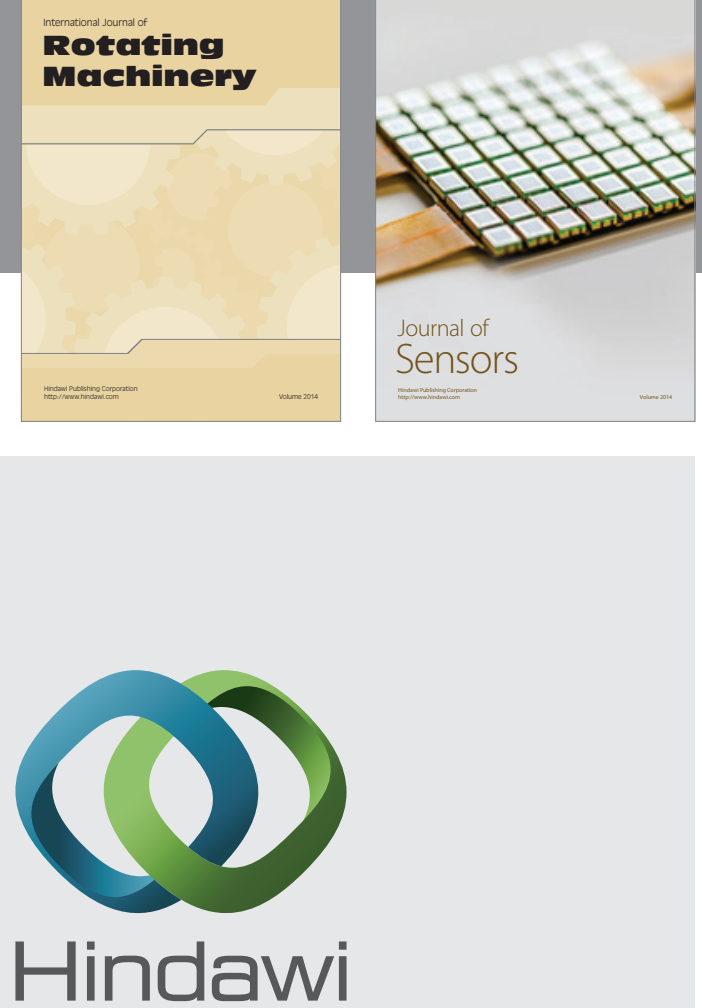

Submit your manuscripts at http://www.hindawi.com
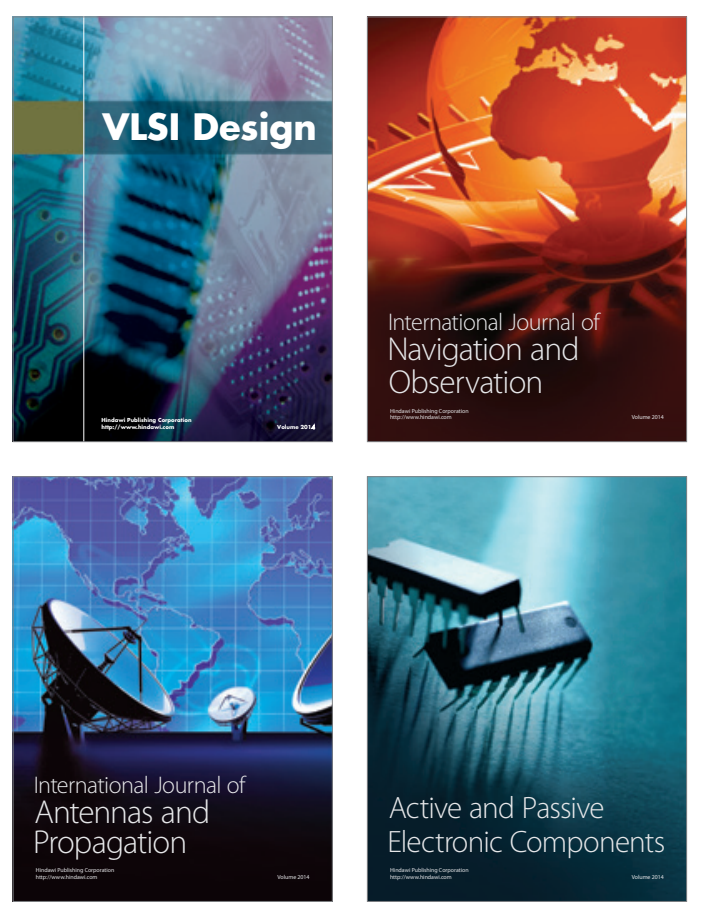
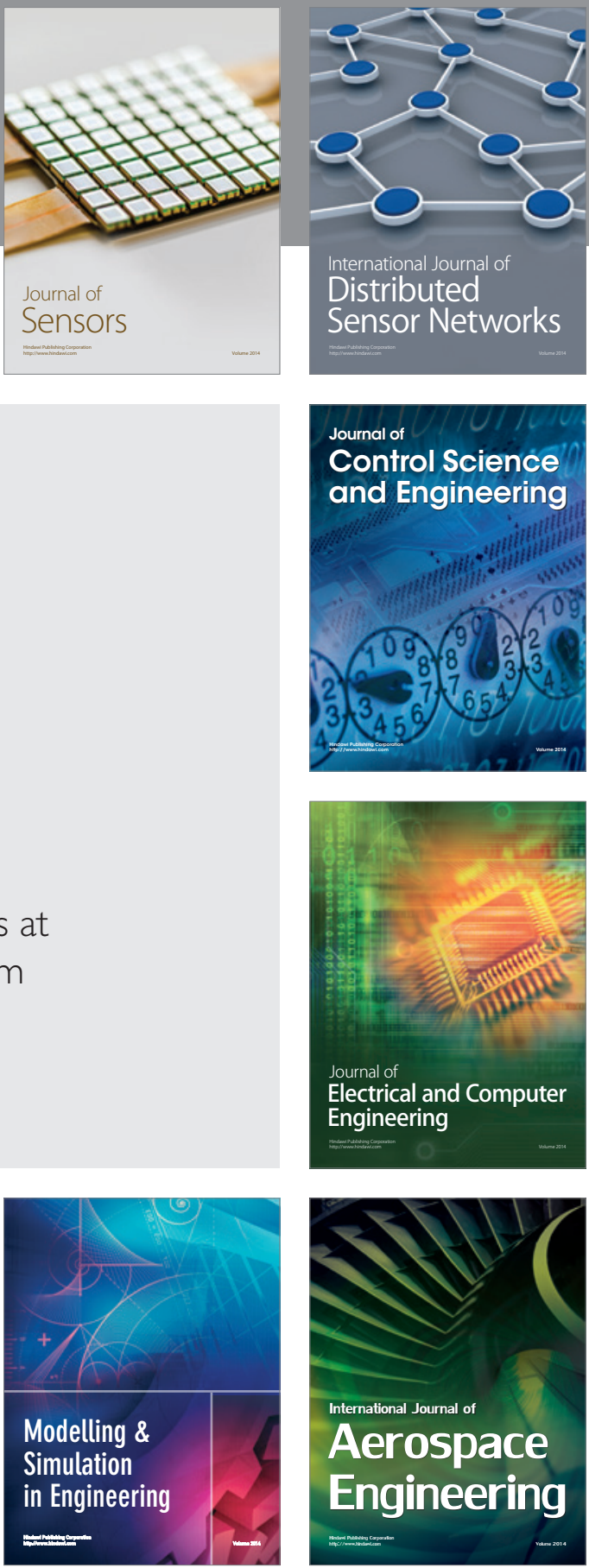

Journal of

Control Science

and Engineering
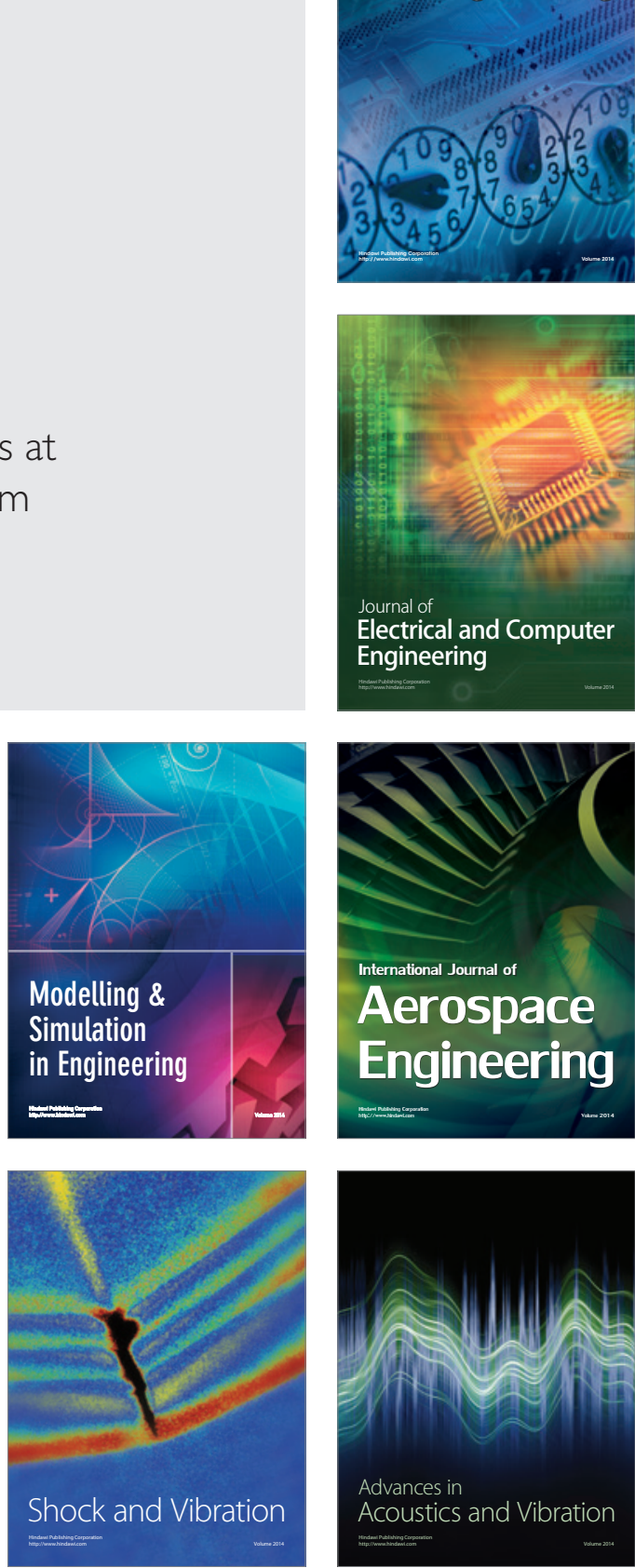